EPJ Web of Conferences 66, 03094 (2014)

DOI: $10.1051 /$ epjconf/ 20146603094

(C) Owned by the authors, published by EDP Sciences, 2014

\title{
Exclusive measurements of nuclear breakup reactions of ${ }^{17} \mathrm{Ne}$
}

F. Wamers ${ }^{1,2,3,4, a}$, J. Marganiec ${ }^{1}$, F. Aksouh ${ }^{4,5}$, Yu. Aksyutina ${ }^{4}$, H. Álvarez-Pol ${ }^{6}$, T. Aumann ${ }^{3,4}$, S. Beceiro-Novo ${ }^{6}$, C.A. Bertulani ${ }^{7}$, K. Boretzky ${ }^{4}$, M.J.G. Borge ${ }^{8}$, M. Chartier ${ }^{9}$, A. Chatillon ${ }^{4}$, L.V. Chulkov ${ }^{4,10}$, D. Cortina-Gil ${ }^{6}$, I.A. Egorova ${ }^{11}$, H. Emling ${ }^{4}$, O. Ershova ${ }^{4,12}$, C. Forssén ${ }^{13}$, L.M. Fraile $^{14}$, H. Fynbo ${ }^{15}$, D. Galaviz ${ }^{8}$, H. Geissel ${ }^{4}$, L.V. Grigorenko ${ }^{4,10,16}$, M. Heil ${ }^{4}$, D.H.H. Hoffmann ${ }^{3}$, J. Hoffmann ${ }^{4}$, H. Johansson ${ }^{13}$, B. Jonson ${ }^{13}$, C. Karagiannis ${ }^{4}$, M. Karakoç ${ }^{7}, 17$, O.A. Kiselev ${ }^{4}$, J.V. Kratz $^{18}$, R. Kulessa ${ }^{19}$, N. Kurz ${ }^{4}$, C. Langer ${ }^{4,12}$, M. Lantz ${ }^{13,20}$, K. Larsson ${ }^{4}$, T. Le Bleis ${ }^{4,21}$, R. Lemmon $^{22}$, Yu.A. Litvinov ${ }^{4}$, K. Mahata ${ }^{4,23}$, C. Müntz ${ }^{12}$, T. Nilsson ${ }^{13}$, C. Nociforo ${ }^{4}$, G. Nyman ${ }^{13}$, W. Ott ${ }^{4}$, V. Panin ${ }^{3,4}$, Yu.L. Parfenova ${ }^{16,24}$, S. Paschalis ${ }^{4,9}$, A. Perea ${ }^{8}$, R. Plag ${ }^{4,12}$, R. Reifarth ${ }^{4,12}$, A. Richter ${ }^{3}$, C. Rodriguez-Tajes ${ }^{6}$, D. Rossi ${ }^{4,18}$, K. Riisager ${ }^{15}$, D. Savran ${ }^{1,2}$, G. Schrieder $^{3}$, N.B. Shul'gina ${ }^{10,13}$, H. Simon ${ }^{4}$, J. Stroth ${ }^{12}$, K. Sümmerer ${ }^{4}$, J. Taylor ${ }^{9}$, O. Tengblad ${ }^{8}$, E. Tengborn ${ }^{13}$, H. Weick $^{4}$, C. Wimmer ${ }^{4,12}$, and M.V. Zhukov ${ }^{13}$

${ }^{1}$ ExtreMe Matter Institute EMMI and Research Division, GSI, Darmstadt, Germany

${ }^{2}$ Frankfurt Institute for Advanced Studies FIAS, Frankfurt am Main, Germany

${ }^{3}$ Institut für Kernphysik, Technische Universität Darmstadt, Darmstadt, Germany

${ }^{4}$ GSI Helmholtzzentrum für Schwerionenforschung GmbH, Darmstadt, Germany

${ }^{5}$ King Saud University, Riyadh, KSA

${ }^{6}$ Universidade de Santiago de Compostela, Santiago de Compostela, Spain

${ }^{7}$ Texas A\&M University-Commerce, Commerce, USA

${ }^{8}$ Instituto de Estructura de la Materia, CSIC, Madrid, Spain

${ }^{9}$ University of Liverpool, Liverpool, UK

${ }^{10}$ Russian Research Center "The Kurchatov Institute", Moscow, Russia

${ }^{11}$ Bogoliubov Laboratory of Theoretical Physics, JINR, Dubna, Russia

${ }^{12}$ Institut für Angewandte Physik, Goethe Universität, Frankfurt am Main, Germany

${ }^{13}$ Chalmers University of Technology, Göteborg, Sweden

${ }^{14}$ Universidad Complutense de Madrid, Marid, Spain

${ }^{15}$ Dept. of Physics and Astronomy, Aarhus University, Aarhus, Denmark

${ }^{16}$ Flerov Laboratory of Nuclear Reactions, JINR, Dubna, Russia

${ }^{17}$ Akdeniz University, Antalya, Turkey

${ }^{18}$ Johannes Gutenberg-Universität Mainz, Mainz, Germany

${ }^{19}$ Jagiellonian University, Krakow, Poland

${ }^{20}$ Uppsala Universitet, Uppsala, Sweden

${ }^{21}$ Technische Universität München, München, Germany

${ }^{22}$ STFC Daresbury Laboratory, Daresbury, UK

${ }^{23}$ Bhabha Atomic Research Centre, Mumbai, India

${ }^{24}$ Institute of Nuclear Physics, Moscow State University, Moscow, Russia

Abstract. We have studied one-proton-removal reactions of about $500 \mathrm{MeV} / \mathrm{u}{ }^{17} \mathrm{Ne}$
beams on a carbon target at the $\mathrm{R}^{3} \mathrm{~B} / \mathrm{LAND}$ setup at GSI by detecting beam-like ${ }^{15} \mathrm{O}-$
p and determining their relative-energy distribution. We exclusively selected the removal

ae-mail: f.wamers@gsi.de

This is an Open Access article distributed under the terms of the Creative Commons Attribution License 2.0, which permits unrestricted use, distribution, and reproduction in any medium, provided the original work is properly cited. 
of a ${ }^{17} \mathrm{Ne}$ halo proton, and the Glauber-model analysis of the ${ }^{16} \mathrm{~F}$ momentum distribution resulted in an $s^{2}$ contribution in the ${ }^{17} \mathrm{Ne}$ ground state of about $40 \%$.

\section{Introduction}

The proton-dripline nucleus ${ }^{17} \mathrm{Ne}$ has raised considerable interest in nuclear physics in the past two decades. While this nucleus also has been studied in the context of nuclear astrophysics [1] and in the search for two-proton radioactivity [2], the focus of the present work has its origin in nuclear-structure physics. The goal is to clarify the ${ }^{17} \mathrm{Ne}$ ground-state structure and its long proclaimed candidacy as a 2proton halo nucleus [3]. Like the famous 2 n-halo nuclei ${ }^{11} \mathrm{Li}$ or ${ }^{6} \mathrm{He},{ }^{17} \mathrm{Ne}$ is a Borromean three-body system. Its binary subsystems (p-p and ${ }^{15} \mathrm{O}-\mathrm{p}$ ) are unbound, and it can be described as an ${ }^{15} \mathrm{O}$ core with two valence protons, predominantly in $\left(1 s_{1 / 2}\right)^{2}$ or $\left(0 d_{5 / 2}\right)^{2}$ configurations. The spin and parity of ${ }^{17} \mathrm{Ne}$ are $J^{\pi}=\frac{1}{2}^{-}$, its half-life for $\beta^{+}$-decay to ${ }^{17} \mathrm{~F}$ is $T_{1 / 2}=109.2 \mathrm{~ms}$, its proton-separation energies are $S_{1 \mathrm{p}}=1479 \mathrm{keV}$ and $S_{2 \mathrm{p}}=943 \mathrm{keV}$, and it does not have any bound excited states. While earlier experiments had already revealed certain indications for the halo characteristics of ${ }^{17} \mathrm{Ne}$ (e.g., an anomalous beta decay branching ratio [4], and large matter radius [5]), the more recent experimental and theoretical studies have focused on the $s^{2}$-weight in the $s^{2} / d^{2}$ configuration mixture of the valence proton pair in the ${ }^{17} \mathrm{Ne}$ ground state. Yet, there is no final conclusion: the three-body model of Grigorenko et al. [6], which is the benchmark for our study, predicts a value of $48 \%$. Kanungo et al. [7] have measured narrow longitudinal-momentum distributions of the ${ }^{15} \mathrm{O}$ core in connection with a large cross section in 2p-removal reactions from ${ }^{17} \mathrm{Ne}$ beams on a Be target. They determined an $s^{2}$-weight of $70 \%$ and concluded to observe a 2-proton halo. However, this experiment was not exclusive to the removal of halo protons, but also includes that of the core protons. Further reported $s^{2}$-weights are $40 \%$ [8], "dominant" [9], and $15 \%$ [10]. Our aim is to provide a conclusive result.

\section{Experimental Technique and Setup}

We report on an experiment performed at GSI in 2007. Secondary ${ }^{17} \mathrm{Ne}$ beams were produced in fragmentation reactions of $(630 \mathrm{MeV} / \mathrm{u})$ primary ${ }^{20} \mathrm{Ne}$ beams impinging on a thick $\left(6.3 \mathrm{~g} / \mathrm{cm}^{2}\right)$ beryllium target at the entrance of the fragment separator (FRS). They were delivered to the experimental setup, impinging on a secondary $\left(370 \mathrm{mg} / \mathrm{cm}^{2}\right)$ carbon target at an energy of about $500 \mathrm{MeV} / \mathrm{u}$ and an intensity of about $10^{4}$ ions/s. In the one-proton knockout reactions induced there, individual protons were instantly removed from the ${ }^{17} \mathrm{Ne}$ projectiles, leading to unbound ${ }^{16} \mathrm{~F}$ decaying in-flight to ${ }^{15} \mathrm{O}+\mathrm{p}$. The ${ }^{16} \mathrm{~F}$ fragments were spectators, thus in the c.m. system carried only the recoil to the intrinsic momentum of the removed proton: $\vec{p}(\mathrm{p})=-\vec{p}\left({ }^{16} \mathrm{~F}\right)$. Correspondingly, their momentum distribution reflects the removed protons' intrinsic momentum. Since the width and shape of these momentum distributions are sensitive to the angular-momentum value of the removed proton, a configuration mixture in the studied nucleus (such as the mentioned $s^{2} / d^{2}$ mixture in ${ }^{17} \mathrm{Ne}$ ) can be decomposed by a fit with momentum distributions calculated in the Eikonal approximation using single-particle wave funtions. At GSI, such reactions were studied using the $\mathrm{R}^{3} \mathrm{~B} / \mathrm{LAND}$ complete-kinematics reaction setup. The ${ }^{17} \mathrm{Ne}$ ions were identified and tracked onto the carbon reaction target, which was surrounded by a box of Si strip detectors and a thick $4 \pi \mathrm{NaI}$ shell segmented into 162 detectors for measuring light recoil particles and gamma rays from excited fragments. ${ }^{15} \mathrm{O}$ fragments and beam-like protons travelling under forward angles were detected in two silicon strip detectors closely behind the target, separated in the ALADIN dipole magnet, and identified and tracked by two fibre detectors and a ToF wall under 16.7 degrees ( ${ }^{15} \mathrm{O}$ fragments), and by two drift chambers and a ToF wall under 31 degrees (protons). 


\section{Analysis and Preliminary Results}

For each one-proton-knockout event, the ${ }^{17} \mathrm{Ne}$ projectile and the ${ }^{15} \mathrm{O}+\mathrm{p}$ ejectiles were identified, tracked, and their four-momenta were reconstructed. Using the invariant-mass technique, the ${ }^{15} \mathrm{O}$ p relative kinetic energy distribution has been determined and is shown in figure $1(\mathrm{a})$, next to the ${ }^{16} \mathrm{~F}$ level scheme (b), and a reaction scheme (c). In figure 1(a), the experimental data (black markers)

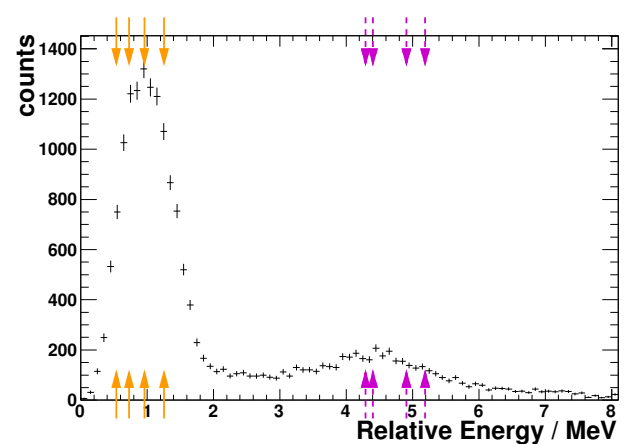

(a)

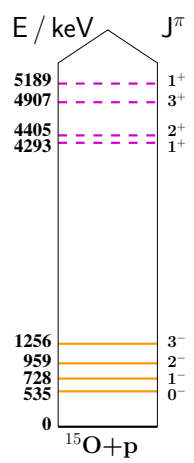

(b)
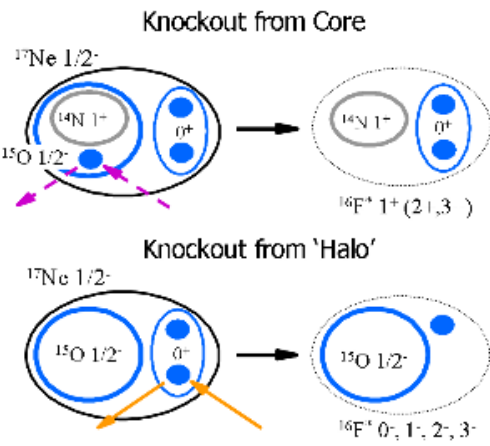

(c)

Figure 1: (a) Measured relative-energy distribution of ${ }^{15} \mathrm{O}-\mathrm{p}$ (black markers), and locations of the ${ }^{16} \mathrm{~F}$ resonances states (coloured arrows). (b) Level scheme of ${ }^{16} \mathrm{~F}$ resonances in terms of the ${ }^{15} \mathrm{O}$-p relative energy [11]. The lowest $0^{-}$state is the ${ }^{16} \mathrm{~F}$ ground state. (c) Proton-knockout processes from ${ }^{17} \mathrm{Ne}$ core and halo states, as depicted in [6].

are shown together with the location (figure 1(b)) of the first four low-lying and next four higherlying ${ }^{16} \mathrm{~F}$ resonances (orange full and purple dashed arrows/lines). The experimental spectrum can be broadly divided into three regions: a narrow low-energy peak between 0 and $2 \mathrm{MeV}$, an intermediate region (2-4 MeV), and a high-lying broad peak (around 4.5 MeV). The low-lying peak is quite well in agreement with the position of the four low-lying negative-parity states in ${ }^{16} \mathrm{~F}$, which can be attributed to the knockout of a halo proton from ${ }^{17} \mathrm{Ne}$. The high-lying broad peak may be attributed to the knockout of a proton from the ${ }^{15} \mathrm{O}$ core of ${ }^{17} \mathrm{Ne}$ leading to population of the positive-parity states in ${ }^{16} \mathrm{~F}$, see figure 1(c) [6]. An exhaustive description of the ${ }^{15} \mathrm{O}$-p relative-energy spectrum is currently ongoing. Detailed Monte-Carlo simulations including our experimental response are being employed, and contributions from competing reaction channels are being evaluated. Nevertheless, gating on the low-energy peak $(<2 \mathrm{MeV})$, yields an exclusive selection of single-proton removal from the ${ }^{17} \mathrm{Ne}$ halo. Figure 2 shows the longitudinal (a) and $\mathrm{x}$-transverse (b) ${ }^{16} \mathrm{~F}$ momentum distributions for events in which a proton was removed from the ${ }^{17} \mathrm{Ne}$ halo. Both distributions have been fitted by a superposition (red full lines) of momentum distributions corresponding to the knockout of either $s$-wave (green dashed lines) or $d$-wave (blue dotted lines) protons using a Glauber-model [12]. The description of the data is very good, and the preliminary analysis yields a value of around $40 \%$ of $s$-wave content in the ${ }^{17} \mathrm{Ne}$ halo.

\section{Summary and Acknowledgements}

We have measured the ${ }^{15} \mathrm{O}$-p relative-energy spectrum and the ${ }^{16} \mathrm{~F}$ momentum distribution following one-proton knockout on ${ }^{17} \mathrm{Ne}$ projectiles. Via a low-energy gate in the ${ }^{15} \mathrm{O}-\mathrm{p} E_{\text {rel }}$ spectrum we can 


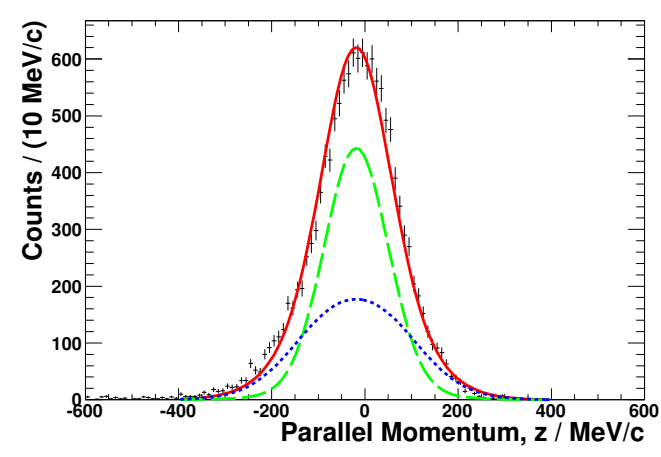

(a) ${ }^{16} \mathrm{~F}$ longitudinal momentum.

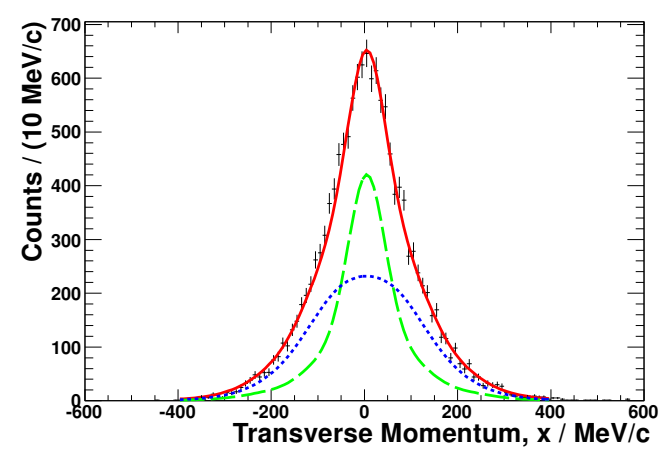

(b) ${ }^{16} \mathrm{~F}$ transverse momentum, $\mathrm{x}$ projection.

Figure 2: Momentum distributions of the ${ }^{16} \mathrm{~F}\left({ }^{15} \mathrm{O}+\mathrm{p}\right)$ system after one-proton knockout from the halo of ${ }^{17} \mathrm{Ne}$. A Glauber-model calculation for proton knockout from single-particle states (green dash: $s$ wave, blue dots: $d$-wave) in ${ }^{17} \mathrm{Ne}$ is used to fit (red full) the experimental data (black markers).

exclusively select the knockout of halo protons, and the Glauber-model analysis of the ${ }^{16} \mathrm{~F}$ recoilmomentum distributions yields an $s$-wave content of about $40 \%$ in the ${ }^{17} \mathrm{Ne}$ halo. The final aspects of the analysis will comprise the setup response, contaminations, and the partial cross sections.

This work was supported by the Alliance Program of the Helmholtz Association (HA216/EMMI), by the German Federal Ministry for Education and Research (BMBF) (project 05P12RDFN8) by HIC for FAIR, by Eurons (European Commission contract no. 506065), and by GSI.

\section{References}

[1] L.V. Grigorenko, K. Langanke, N.B. Shul' gina, M.V. Zhukov, Physics Letters B 641, 254 (2006)

[2] M.J. Chromik, P.G. Thirolf, M. Thoennessen, B.A. Brown, T. Davinson, D. Gassmann, P. Heckman, J. Prisciandaro, P. Reiter, E. Tryggestad et al., Physical Review C 66, 024313 (2002)

[3] M.V. Zhukov, I.J. Thompson, Physical Review C 52, 3505 (1995)

[4] M.J.G. Borge, J. Deding, P.G. Hansen, B. Jonson, G. Martinez-Pinedo, P. Møller, G. Nyman, A. Poves, A. Richter, K. Riisager et al., Physics Letters B 317, 25 (1993)

[5] A. Ozawa, T. Kobayashi, H. Sato, D. Hirata, I. Tanihata, O. Yamakawa, K. Omata, K. Sugimoto, D. Olson, W. Christie et al., Physics Letters B 334, 18 (1994)

[6] L.V. Grigorenko, Y.L. Parfenova, M.V. Zhukov, Physical Review C 71, 051604 (2005)

[7] R. Kanungo, M. Chiba, B. Abu-Ibrahim, S. Adhikari, D. Q. Fang, N. Iwasa, K. Kimura, K. Maeda, S. Nishimura, T. Ohnishi et al., European Physics Journal A 25, 327 (2005)

[8] W. Geithner, T. Neff, G. Audi, K. Blaum, P. Delahaye, H. Feldmeier, S. George, C. Guénaut, F. Herfurth, A. Herlert et al., Physical Review Letters 101, 252502 (2008)

[9] K. Tanaka, M. Fukuda, M. Mihara, M. Takechi, D. Nishimura, T. Chinda, T. Sumikama, S. Kudo, K. Matsuta, T. Minamisono et al., Physical Review C 82, 044309 (2010)

[10] T. Oishi, K. Hagino, H. Sagawa, Phys. Rev. C 82, 024315 (2010)

[11] D.R. Tilley, H.R. Weller, C.M. Cheves, Nuclear Physics A 564, 1 (1993)

[12] M. Karakoç, A. Banu, C.A. Bertulani, L. Trache, Phys. Rev. C 87, 024607 (2013) 